\title{
Produtos naturais utilizados para tratamento de asma em crianças residentes na cidade de Salvador-BA, Brasil
}

\author{
Ryan dos S. Costa, ${ }^{1}$ Tamires C. Brasil,, ${ }^{1}$ Carla de J. Santos, ${ }^{1}$ Djanilson B Santos, ${ }^{2}$ \\ Maurício L Barreto, ${ }^{3}$ Neuza M. Alcântara Neves, ${ }^{1}$ Camila A. V. de Figueiredo ${ }^{* 1}$
}

\author{
${ }^{1}$ Instituto de Ciências da Saúde, Universidade Federal da Bahia, Avenida Reitor Miguel Calmon s/n, Vale do Canela, \\ 40140-100 Salvador-BA, Brasil, \\ ${ }^{2}$ Centro de Ciências da Saúde, Universidade Federal do Recôncavo da Bahia, Rua do Cajueiro, 44570-000 Santo \\ Antônio de Jesus-BA, Brasil, \\ ${ }^{3}$ Instituto de Saúde Coletiva, Universidade Federal da Bahia, Rua Basílio da Gama s/n, Campus Universitário \\ Canela, 40110-040 Salvador-BA, Brasil.
}

\begin{abstract}
RESUMO: O conhecimento popular é o passo inicial para a investigação científica de atividades terapêuticas de remédios caseiros. Diversas patologias podem ser tratadas ou amenizadas através de preparações de origem natural e muitos fármacos disponíveis são oriundos de fontes naturais. Este trabalho tem como objetivo avaliar o uso de remédios caseiros derivados de plantas para tratamento de asma em crianças residentes no município de Salvador. Os dados foram obtidos a partir de estudo realizado em Salvador sobre fatores de risco, uso de medicações e vias imunológicas relacionadas à asma em crianças. Foram calculadas as freqüências de uso de preparações caseiras para tratamento de asma por esta população e realizado uma revisão bibliográfica sobre os efeitos das plantas mais usadas. Dentre as espécies mais citadas, destacam-se o Allium sativum (alho) que teve a maior freqüência de utilização na preparação dos remédios caseiros (25\%), seguido da Allium cepa (cebola, 19,74\%). Após a revisão crítica de literatura, constatou-se que a maioria das espécies é utilizada com base em relatos fundamentados no saber popular, sendo assim carente de evidências científicas para as atividades farmacoterapêuticas esperadas. Neste sentido, há necessidade de mais estudos farmacológicos para comprovação das atividades terapêuticas peculiares a cada produto de origem natural bem como para avaliar possíveis efeitos tóxicos destes produtos.
\end{abstract}

Unitermos: medicina popular, asma, etnofarmacologia.

ABSTRACT: "Natural products used for asthma treatment in children living in SalvadorBA, Brazil". The popular knowledge is the initial step for the scientific inquiry of therapeutical activities of herb-based remedies. Several pathologies can be treated or brightened up through this kind of preparations and also many of the available drugs in the market have natural sources. The objective of this work was to evaluate the use of herb-based remedies for treatment of asthma in children in the city of Salvador. Data were collected by a standard questionnaire during a transversal study carried out in Salvador on risk factors, use of medications and immunological pathways involved in asthma. Among the most frequently mentioned species, the Allium sativum had the highest frequency of use in the preparation of home remedies $(25 \%)$, followed by the Allium cepa (19.74\%). The literature review showed that the majority of the species is empirically used based on popular knowledge and lacks on scientific evidences that prove their pharmacotherapeutic activities and safety for human use. In this way, this work not only new species unexplored in the context of anti-asthmatic drugs but it also highlights the need for new pharmacological studies in order to identify and prove the popular use of herb-based remedies.

Keywords: medicine knowledge, asthma; ethnopharmacology.

\section{INTRODUÇ̃̃O}

A asma é uma doença inflamatória crônica caracterizada por hiperresponsividade das vias aéreas inferiores e por limitação variável ao fluxo aéreo, manifestando-se clinicamente por episódios recorrentes de sibilância, dispnéia e tosse (O’Byrne, 2009). A asma constitui um problema importante de saúde pública, uma vez que tem alta prevalência, expõe o paciente a recorrentes hospitalizações e representa uma elevada carga econômica para o indivíduo, a sociedade e o governo (Lee, 2010; Franco et al., 2009). Alta prevalência de asma tem sido 
observado em diferentes cidades brasileiras, incluindo Salvador (Solé et al., 2007).

Atualmente, a terapia disponível para a asma é baseada na broncodilatação, utilizando agonistas adrenérgicos ou antagonistas colinérgicos; ou baseada na supressão da inflamação, sendo os glicocorticóides as principais drogas disponíveis. Essas estratégias auxiliam no controle dos sintomas da asma, no entanto apresentam diversos efeitos colaterais e, principalmente, não levam à cura. (Cohn et al., 2004; Walsh, 2005).

Devido às restrições impostas pelas atuais terapias disponíveis, a alergia é relatada como a segunda patologia em que a população mundial dá preferência ao uso de medicamentos complementares (Bielory, 2004). Na Alemanha ocidental, por exemplo, $30 \%$ dos portadores de alergias utilizam medicamentos alternativos, sendo os fitoterápicos os mais importantes deles (Schafer, 2004).

Anecessidade de desenvolver medidas eficazes de controle para a asma resultou na exploração destas terapias alternativas, incluindo remédios de ervas tradicionalmente utilizadas em países do velho mundo (Kurup \& Barrios, 2008). Sendo assim, a etnofarmacologia destaca-se como poderosa ferramenta para obtenção de informações necessárias ao desenvolvimento de novos fármacos de interesse médico e farmacêutico a partir de produtos naturais, de modo que a flora brasileira, e em particular a nordestina, apresenta-se como importante fonte de produtos naturais a serem estudados (Albuquerque \& Hanazaki, 2006).

Outro aspecto importante a ser considerado quanto à etnofarmacologia é a busca por informações sobre a eficácia e segurança da utilização popular dos produtos naturais, principalmente devido às possíveis interações com outros produtos seja de origem natural, seja sintético, utilizada concomitantemente pelo paciente

Desta forma, o presente estudo teve por objetivo realizar um levantamento dos produtos naturais utilizados para tratamento de asma em crianças residentes no município de Salvador, bem como confrontar o conhecimento tradicional com as atividades terapêuticas já descritas na literatura para as espécies vegetais com maior freqüência de utilização pela população estudada.

\section{MATERIAIS E MÉTODOS}

O presente trabalho foi desenvolvido a partir da utilização das informações oriundas de uma coorte em curso em Salvador para o estudo dos fatores de risco para asma, perfil de imunológico e tratamento utilizados (Barreto et al., 2006). O protocolo foi aprovado pelo CONEP (Conselho Nacional de Ética em Pesquisa) e consentimento informado foi obtido por escrito de todos os responsáveis pelas crianças estudadas (número do processo 003-05/CEP-ISC).

$\mathrm{O}$ estudo que serviu de fundamento para a realização deste trabalho envolveu 1372 crianças com idades compreendidas entre 4-11 anos em 2005 quando os dados analisados foram coletados. Os dados sobre sintomas de asma foram obtidos usando um questionário do ISAAC fase II, traduzido para o português e os dados sobre medicações utilizadas para o tratamento de asma foram obtidos com o emprego de um questionário previamente validado (Santos et al., 2009). Além disso, foi aplicado o termo de consentimento livre e esclarecido aos pais ou responsável legal da criança (Barreto et al., 2006).

As perguntas constantes no questionário relativas ao uso de preparações caseiras foram:

1. $\mathrm{O}$ (a) senhor(a) costuma dar remédio caseiro à criança como chás, xaropes e soro?

2. Alguma vez na vida a criança usou algum remédio caseiro para asma?

3. Nos últimos 12 meses seu filho(a) usou algum remédio como comprimidos ou xaropes, para chiado, piado no peito ou asma? Qual?

A partir dos dados obtidos foi possível estabelecer a freqüência de utilização de remédios caseiros para patologias em geral e, especificamente para a asma. Também foi possível determinar quais foram os produtos naturais utilizados, bem como a freqüência de utilização destes.

Os produtos naturais mais citados para o tratamento de asma foram, então, submetidos à revisão da literatura, de modo a confrontar a utilização popular com informações científicas referentes aos mesmos. Para isso, foram consultadas as bases de dados Pubmed, Highwire e o Scielo, utilizando como marcadores os termos "asthma AND herbs", "asthma AND medicinal plants", bem como o termo asthma em associação com os nomes científicos das espécies vegetais estudadas. O critério de seleção dos artigos foi que esses apresentassem estudo in vitro ou in vivo avaliando as propriedades farmacológicas, a eficácia e/ou a segurança das espécies investigadas.

\section{RESULTADOS}

A partir da resposta para a questão " $\mathrm{O}(\mathrm{a})$ senhor(a) costuma dar remédio caseiro à criança como chás, xaropes e soro?" foi obtida a freqüência de $79,6 \%(n=1092)$ de utilização de remédios caseiros pela população infantil de um modo geral (Figura 1).

No entanto, quando refere-se à utilização de remédios caseiros especificamente para o tratamento de asma, apenas $121(8,9 \%)$ entrevistados responderam positivamente a questão "Alguma vez na vida a criança usou algum remédio caseiro para asma?" (Figura 2).

A relação dos produtos naturais mais utilizados para o tratamento de crianças com asma pela população estudada foi obtido a partir das respostas à questão "Nos últimos 12 meses seu filho(a) usou algum remédio como comprimidos, xaropes, para chiado, piado no peito ou asma? Qual?" (Tabela 1). 


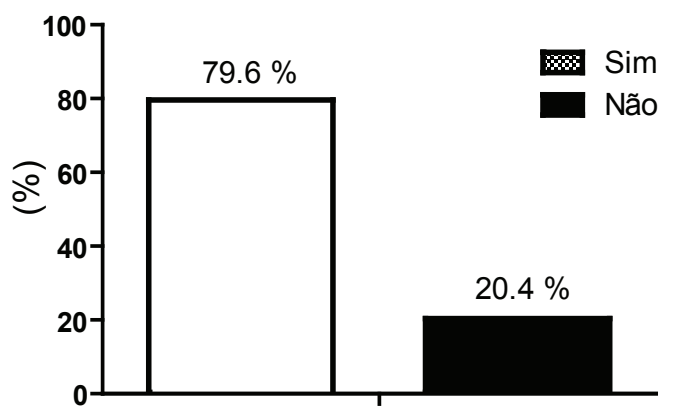

Figura 1. Freqüência do uso de remédios caseiros pela população estudada, na cidade de Salvador-BA, em 2005. As barras representam as porcentagens de pessoas entrevistadas que responderam sim ou não à pergunta " $\mathrm{O}(\mathrm{a})$ senhor(a) costuma dar remédio caseiro à criança como chás, xaropes e soro?”

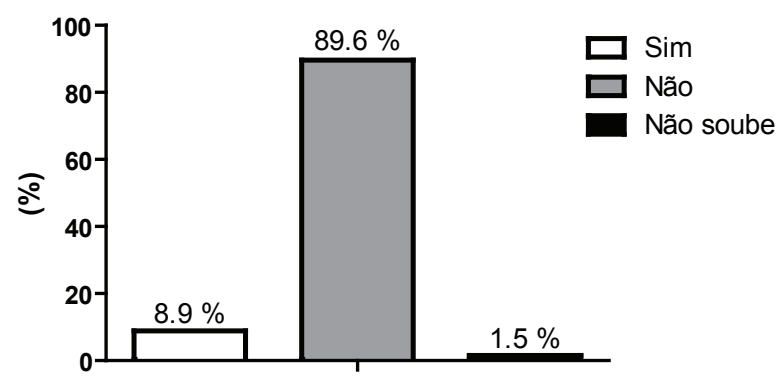

Figura 2. Freqüência de remédios caseiros utilizados para o tratamento de crianças com asma, na cidade de Salvador-BA, em 2005. As barras representam as porcentagens de pessoas entrevistadas que responderam sim, não ou não souberam à pergunta "Alguma vez na vida a criança usou algum remédio caseiro para asma?"

Tabela 1. Relação dos produtos de origem natural com uso relatado pelos entrevistados para o tratamento de asma em crianças. Para cada produto é atribuído o possível binômio científico, bem como a respectiva freqüência de citação pelos entrevistados (\%).

\begin{tabular}{|c|c|c|c|}
\hline Produto & Possível binômio científico & (n) & Freqüência $(\%)$ \\
\hline Mel & - & 26 & 34,21 \\
\hline Alho & Allium sativum L., Amaryllidaceae & 19 & 25 \\
\hline Cebola & Allium cepa L., Amaryllidaceae & 15 & 19,74 \\
\hline Beterraba & Beta vulgaris L., Chenopodiaceae & 11 & 14,47 \\
\hline Limão & Citrus limoniu, Rutaceae & 10 & 13,16 \\
\hline Capim-santo & Cymbopogon citratus Poaceae & 7 & 9,21 \\
\hline Maria preta & Solanum americanum Mill., Solanaceae & 7 & 9,21 \\
\hline Sabugueiro & Sambucus sp., Adoxaceae & 7 & 9,21 \\
\hline Abacaxi & Ananas comosus (L.) Merr., Bromeliaceae & 4 & 5,26 \\
\hline Cravo & Eugenia caryophyllata L., Myrtaceae & 4 & 5,26 \\
\hline Quioio & Ocimum gratissimum L., Lamiaceae & 4 & 5,26 \\
\hline Pitanga & Eugenia uniflora L., Myrtaceae & 3 & 3,95 \\
\hline Poejo & Mentha pulegium L., Lamiaceae & 3 & 3,95 \\
\hline Agrião & Rorippa nasturtium-aquaticum (L.) Hayek, Brassicaceae & 2 & 2,63 \\
\hline Alevante & Achillea millefolium L., Asteraceae & 2 & 2,63 \\
\hline Banana & Musa acuminata Colla, Musaceae & 2 & 2,63 \\
\hline Hortelã & Mentha sp., Lamiaceae & 2 & 2,63 \\
\hline Amburana & Torresea cearensis L. Fabaceae & 1 & 1,31 \\
\hline Cidreira & Melissa officinalis L., Lamiaceae & 1 & 1,31 \\
\hline Folha da Costa & Kalanchoe pinnata (Lam.) Pers. Crassulaceae & 1 & 1,31 \\
\hline Laranja & Citrus sinensis (L.) Osbeck, Rutaceae & 1 & 1,31 \\
\hline Pimentão & Capsicum annuиm L., Solanaceae & 1 & 1,31 \\
\hline Própolis & - & 1 & 1,31 \\
\hline Rabanete & Raphanus sativus L., Brassicaceae & 1 & 1,31 \\
\hline Sena & Senna alexandrina Mill., Fabaceae & 1 & 1,31 \\
\hline \multicolumn{2}{|l|}{ Total } & 136 & \\
\hline
\end{tabular}


$\mathrm{O}$ alho (Allium sativum L.), teve a maior freqüência de utilização nas preparações caseiras $(25 \%)$, seguido da cebola (Allium cepa L.) $(19,74 \%)$, beterraba (Beta vulgaris L.) (14,47\%), limão (Citrus limonium) (13,16\%), capim-santo (Cymbopogon citratus) (9,21\%), maria-preta (Solanum americanum Mill.) (9,21\%), sabugueiro (Sambucus sp.) (9,21\%) e o quioio (Ocimum gratissimum L.) $(5,26 \%)$. O único produto natural citado que não apresenta origem vegetal foi o mel, com freqüência de $34,21 \%$.

\section{DISCUSSÃO}

O estudo etnofarmacológico contribui para a bioprospecção e descoberta de novos fármacos, bem como para a preservação da biodiversidade e promoção do uso racional das plantas em combinação com os fármacos disponíveis (Albuquerque \& Hanazaki, 2006). Deste modo, torna-se uma importante ferramenta para a exploração científica do conhecimento popular.

Medicinas alternativas e complementares (MAC), dentre elas os produtos naturais, são utilizadas por cerca de $80 \%$ da população mundial, o que as tornam um importante componente do sistema de saúde de diversos países. Das patologias tratadas, as alergias merecem destaque pelo crescente número de terapias baseadas nas MAC, existindo uma diversidade de estudos demonstrando a influência desta prática no sistema imunológico do paciente alérgico (Mainardi, 2009). Atualmente, a freqüência de indivíduos menores de dezoito anos alérgicos que já fizeram uso de alguma dessas terapias corresponde a mais de 5\% da população mundial (Engler et al., 2009).

No presente estudo com crianças da cidade de Salvador, a freqüência de utilização de produtos naturais para o tratamento de patologias em geral foi compatível com estudos prévios, contabilizando $79,6 \%$ da população estudada. Destas, $8,9 \%$ fizeram uso de produtos naturais especificamente para o tratamento de asma.

A prevalência de asma na população estudada foi de $22 \%$ (dados não mostrados) sendo que $40,5 \%$ destas crianças asmáticas fizeram uso de produtos naturais para o tratamento desta patologia. Freqüências semelhantes ou mais elevadas também foram observadas em outros estudos, como o realizado por Bearison et al. (2002) numa comunidade hispânica, onde $72 \%$ das mães entrevistadas referiram usar produtos naturais para prevenir asma em crianças.

A utilização de produtos naturais para o tratamento de indivíduos asmáticos é realizada, possivelmente, em associação com drogas padrão para a asma, como broncodilatadores, agonistas adrenérgicos $\beta 2$ e corticosteróides. Embora essa associação possa ser benéfica para a terapêutica do paciente, a literatura carece de estudos científicos avaliando a eficácia e segurança da interação destes produtos.

No entanto, diversos estudos pré-clinicos e clínicos têm sido conduzidos avaliando as atividades de produtos naturais no tratamento da asma, sendo que estudos já demonstraram que algumas plantas medicinais possuem propriedades antiinflamatórias, por exemplo, que podem ser usadas na supressão da resposta imunológica exacerbada em pacientes asmáticos (Clement et al., 2005).

Neste trabalho, os entrevistados citaram vinte e cinco produtos naturais diferentes que foram utilizados para o tratamento de crianças com asma. Dentre os produtos vegetais citados, os que possuem alguma fundamentação científica foram o alho, a cebola, o capim-santo, o limão, o sabugueiro, a maria-preta e o quioio.

$\mathrm{O}$ alho (Allium sativum L.) tem sido utilizado na saúde pública há anos em alguns tratamentos (Semra et al., 2007) dentre eles para asma e outras desordens respiratórias (Rivlin, 2001; Moyers, 1996). Os mais importantes compostos químicos provenientes do alho são os derivados de enxofre (sulfatados), com destaque para a alicina que apresenta propriedades antimicrobianas, antifúngicas, antiparasitárias e anti-carcinogênica (Semra et al., 2007).

Em relação à propriedade imunorregulatória do Allium sativum, estudos em camundongos tem mostrado seu potencial em estimular a produção de citocinas do perfil Th1 e diminuir os níveis de IgE e IgG1 específicos e de IFN; sugerindo assim que essa espécie possui potencial de atenuar a resposta inflamatória nas alergias (Zare et al., 2008)

A cebola (Allium cepa L.) foi o segundo produto de origem vegetal mais citado para o tratamento da asma no levantamento realizado, representando $19,74 \%$ na utilização. Esses dados são confirmados na literatura científica que demonstra o efeito anti-alérgico dessa espécie. Estudo etnobotânico realizado numa comunidade hispânica demonstrou que tanto o alho quanto a cebola tiveram utilização de $40 \%$ em preparações para terapia da asma (Bearison et al., 2002). Allium cepa L., possui propriedades antimicrobianas, antitrombótica, antitumoral, hipoglicemiante e antialérgica. Entre os compostos com atividade broncodilatadora, destacam-se os isotiocianatos, os quais inibem as enzimas 5-lipoxigenase e a cicloxigenase in vitro e inibe, em uma forma dose-dependente, a obstrução brônquica. Os extratos etanólico e clorofórmico têm demonstrado atividade broncodilatadora em humanos, não apresentando efeito anti-histamínico e anticolinérgico o que sugere uma regulação do sistema imunológico. (Tôrres et al, 2005; Dorsch et al., 1984) Desta forma, a cebola poderia ser usada como recurso terapêutico embora novos estudos sejam necessários para investigar o exato mecanismo de ação responsável por tais atividades farmacológicas.

O limão (Citrus limonium) foi citado por 13,61\% dos entrevistados. A asma sintomática em adultos está associada a uma baixa ingestão de frutas, que contém, por exemplo, moléculas antioxidantes, tal como a vitamina 
C, presente no Citrus limonium. Então, a dieta alimentar pobre em antioxidantes pode ser um fator de risco para o desenvolvimento de asma (Patel et al., 2006). No entanto, outro estudo não demonstrou associação entre o consumo de frutas cítricas com a asma (Tabak et al., 2006).

O capim-santo, também conhecido como capimlimão ou erva-cidreira (Cymbopogon citratus) foi citado por $9,21 \%$ dos entrevistados na utilização para o tratamento da asma. A medicina popular utiliza a infusão, preparada a partir de suas folhas, como calmante, analgésico, em dores abdominais e de cabeça, antifebril, antireumático, carminativo, antitussígeno, diurético, diaforético e em distúrbios digestivos. Essas propriedades são devidas, principalmente, ao óleo volátil, componente majoritário da planta que é basicamente constituído de alfacitral (geranial) e beta-citral (neral). Outro componente da planta é o mirceno (7-metil-3-metileno-1,6-octadiene), já reconhecido por sua ação analgésica periférica. (Schuck et al., 2001; Zamith et al., 1993) Na literatura existem relatos da atividade antiinflamatória do citral e da planta (Lee et al., 2008; Carbajal et al., 1989), porém, não foi encontrado nenhum trabalho referindo a imunomodulação dessa espécie.

O sabugueiro (Sambucus sp.) é utilizado na forma de decocção ou infusão para resfriados e asma (Guarrera, 2005) e foi citado por $9,21 \%$ dos entrevistados na utilização para o tratamento da asma. Esta espécie é de origem européia e suas flores são comercializadas in natura sob o nome farmacopéico Sambuci flos. São também usadas como diuréticas, antipiréticas, antiinflamatórias, laxativas leves e no tratamento de doenças do aparelho respiratório (Scopel et al., 2007).

A maria-preta (Solanum americanum Mill.) foi citada por $9,21 \%$ dos entrevistados na utilização para o tratamento da asma. Solanum americanum Mill. apresenta uma composição química complexa e pouco estudada contendo alcaloides. Essa espécie é usada para tratar anemia, condições dermatomucosas, além de possuir propriedade calmante, depurativa, antiinflamatória, emoliente, febrífuga e sedativa. (Villatoro, 2004). A espécie de mesmo gênero, Solanum melongena, tem efeito broncoespamogênico, ao invés de broncoespamolítico, provavelmente por estimulação de receptores muscarínicos (Mans et al., 2004).

O quioio (Ocimum gratissimum L.) foi citado por $5,26 \%$ dos entrevistados na utilização para o tratamento da asma. É constituído por um fitoquímico polifenólico, encontrado na família Lamiaceae, o ácido rosmarínico. Este, também encontrado no extrato da espécie Perilla frutescens, pode ser uma intervenção efetiva para pacientes com rinoconjuntivite alérgica sazonal leve, através da inibição da inflamação dependente de leucócitos polimorfonucleares (Takano et al., 2004). O ácido rosmarínico previne a inflamação eosinofílica de vias aéreas induzida por alérgeno de ácaro da poeira domiciliar em camundongos que está associado com a inibição do aumento local da expressão de citocinas Th2 e quimiocinas e da inibição da produção aumentada de imunoglobulina alérgeno-específica (Sanbongi et al., 2004). Todos estes achados na literatura corroboram para um possível efeito imunomodulador do Ocimum gratissimum L. e seu constituinte o ácido rosmarínico, mecanismo pelo qual poderia explicar seu uso popular para afecções alérgicas.

Diante do exposto foi constatado que a maioria das espécies utilizada empiricamente por esta população, com base em relatos fundamentados no "saber popular", é carente de evidências científicas que comprovem as atividades farmacoterapêuticas esperadas. Sendo assim, há necessidade de mais estudos farmacológicos para comprovação das atividades terapêuticas peculiares a cada produto de origem natural e seus possíveis efeitos tóxicos.

\section{REFERÊNCIAS}

Albuquerque UP, Hanazaki N 2006. As pesquisas etnodirigidas na descoberta de novos fármacos de interesse médico e farmacêutico: fragilidade e perspectivas. Rev Bras Farmacogn 16: 678-689.

Barreto ML, Cunha SS, Alcântara-Neves N, Carvalho LP, Cruz AA, Stein RT, Genser B, Cooper PJ, Rodrigues LC 2006. Risk factors and immunological pathways for asthma and other allergic diseases in children: background and methodology of a longitudinal study in a large urban center in Northeastern Brazil (Salvador-SCAALA study). BMC Pulm Med 6: 1-10.

Bearison DJ, Minian N, Granowetter L 2002. Medical management of asthma and folk medicine in a hispanic community. J Pediatr Psychol 27: 385-392.

Bielory L 2004. Complementary and alternative interventions in asthma, allergy, and immunology. Ann Allergy Asthma Immunol 2: 45-54

Carbajal D, Casaco A, Arruzazabala L, Gonzalez R, Tolon Z. 1989. Pharmacological study of Cymbopogon citratus leaves. J Ethnopharmacol 25: 103-107.

Clement YN, Williams AF, Aranda D, Chase R, Watson N, Mohammed R, Stubbs O, Williamson D 2005. Medicinal herb use among asthmatic patients attending a specialty care facility in Trinidad. BMC Complement Altern Med 5: 1-8.

Cohn L, Jack EA, Chupp GL 2004. Asthma: mechanisms of disease persistence and progression. Annu Rev Immunol 22: 789-815.

Dorsch W, Adam O, Weber J, Ziegeltrum T 1984. Antiasthmatic effects of onion extracts-detection of benzyl- and other isothiocyanates (mustard oils) as antiasthmatic compounds of plant origin. Eur J Pharmacol 107: 1724.

Engler RJ, With CM, Gregory PJ, Jellin JM 2009. Complementary and alternative medicine for the allergist-immunologist: Where do I start? J Allergy Clin Immunol 123: 309-316..

Franco R, Nascimento HF, Cruz AA, Santos AC, Souza-Machado C, Ponte EV, Souza-Machado A, Rodrigues LC, Barreto ML2009. The economic impact of severe asthma to lowincome families. Allergy 64: 478-83.

Guarrera PM 2005. Traditional phytotherapy in Central Italy (Marche, Abruzzo, and Latium). Fitoterapia 76: 1-25. 
Kurup VP, Barrios CS 2008. Immunomodulatory effects of curcumin in allergy. Mol Nutr Food Res 52: 1031-1039.

Lee HJ, Jeong HS, Kim DJ, Noh YH, Yuk DY, Hong JT 2008. Inhibitory effect of citral on $\mathrm{NO}$ production by suppression of iNOS expression and NF-kappa B activation in RAW264.7 cells. Arch Pharm Res 31: 342-349.

Lee S 2010. Prevalence of childhood asthma in Korea: International study of asthma and allergies in childhood. Allergy Asthma Immunol Res 2: 61-64.

Mainardi T, Kapoor S, Bielory L 2009. Complementary and alternative medicine: herbs, phytochemicals and vitamins and their immunologic effects. J Allergy Clin Immun 123: 283-294

Mans DR, Toelsie J, Mohan S, Jurgens S, Muhringen M, Illes S, Macnack R, Bipat R 2004. Spasmogenic effect of a Solanum melongena leaf extract on guinea pig tracheal chains and its possible mechanism(s). J Ethnopharmacol 95: 329-333.

Moyers S 1996. Garlic in health, history and world cuisine. Suncoast Press 3: 1-36.

O'Byrne PM 2009. Allergen-induced airway inflammation and its therapeutic intervention. Allergy Asthma Immunol Res 1: 3-9.

Patel BD, Welch AA, Bingham SA, Luben RN, Day NE, Khaw KT, Lomas DA, Wareham NJ 2006. Dietary antioxidants and asthma in adults. Thorax 61: 388-393.

Rivlin RS 2001. Historical perspective on the use of garlic. $J$ Nutr 131: 951-954.

Sanbongi C, Takano H, Osakabe N, Sasa N, Natsume M, Yanagisawa R, Inoue KI, Sadakane K, Ichinose $\mathrm{T}$, Yoshikawa T 2004. Rosmarinic acid in perilla extract inhibits allergic inflammation induced by mite allergen, in a mouse model. Clin Exp Allergy 34: 971-977.

Santos DB, Barreto ML, Coelho HLL 2009. Utilização de medicamentos e fatores associados entre crianças residentes em áreas pobres. Rev Saude Publica 43: 768778.

Schafer T 2004. Epidemiology of complementary alternative medicine for asthma and allergy in Europe and Germany. Ann Allergy Asthma Immunol. 2: 5-10.

Schuck VJA, Fratini M, Rauber CS, Henriques A, Schapoval EES 2001. Avaliação da atividade antimicrobiana de Cymbopogon citratus. Rev Bras Cienc Farm 37: 45-49.

ScopelM,Nunes, E, Vignoli-Silva, M, Vendruscolo, GS, Henriques, AT, Mentz, LA 2007. Caracterização farmacobotânica das espécies de Sambucus (Caprifoliaceae) utilizadas como medicinais no Brasil. Parte I. Sambucus nigra L. Rev Bras Farmacogn 17: 249-261

Semra Ö, Zeynep S, Serpil D, Fatih O, Atalay S 2007. Sarimsak (Allium Sativum) Özütü Skolosidal Ajan Olarak Kullanılabilir Mi? Turkiye Parazitol Derg 31: 318-321.

Solé D, Melo KC, Camelo-Nunes IC, Freitas LS, Britto M, Rosário NA, Jones M, Fischer GB, Naspitz CK.2007. Changes in the prevalence of asthma and allergic diseases among Brazilian schoolchildren (13-14 years old): comparison between ISAAC phases one and three. J Trop Pediatr 53: 13-21.

Tabak C, Wijga AH, de Meer G, Janssen NA, Brunekreef B, Smit HA 2006. Diet and asthma in Dutch school children (ISAAC-2). Thorax 61: 1048-1053.

Takano H, Osakabe N, Sanbongi C, Yanagisawa R, Inoue K, Yasuda A, Natsume M, Baba S, Ichiishi E, Yoshikawa T 2004. Extract of Perilla frutescens enriched for rosmarinic acid, a polyphenolic phytochemical, inhibits seasonal allergic rhinoconjunctivitis in humans. Exp Biol Med 229: 247-254.

Tôrres AR, Oliveira RAG, Diniz MFFM, Araújo EC 2005. Estudo sobre o uso de plantas medicinais em crianças hospitalizadas da cidade de João Pessoa: riscos e benefícios. Rev Bras Farmacogn 15: 373-380.

Villatoro MCP 2004. Inhibicion del crecimiento de gardnerella vaginalis por seis plantas de uso medicinal de la flora suroccidental guatemalteca. Guatemala, Tese de doutorado, Facultad de Ciencias Quimicas y Farmácia, Universidad de San Carlos de Guatemala.

Walsh GM 2005. Novel therapies for asthma-advances and problems. Curr Pharm Design 11: 3027-3038.

Zamith HP, Vidal MN, Speit G, Paumgartten FJ.al 1993. Absence of genotoxic activity of beta-myrcene in the in vivo cytogenetic bone marrow assay. Braz J Med Biol Res 26: 93-98.

Zare A, Farzaneh P, Pourpak Z, Zahedi F, Moin M, Shahabi S, Hassan ZM 2008. Purified aged garlic extract modulates allergic airway inflammation in BALB/c mice. Iran $J$ Allergy Asthma Immunol 7: 133-141. 